\title{
An Experience of Short-Term Results of Laparoscopic Inguinal Hernioplasty Using 3D Mesh in a Developing Country
}

\author{
Iqbal Saleem Mir*, Alfer Ah Nafae, Aijaz Ahmed Malyar, Muntakhab Nafae, Yawar Watali, \\ Mudasir Farooq, Shahnawaz Bashir Bhat, Sheikh Viqar
}

The Post-Graduate Department of Surgery, Govt Medical College, Srinagar, India

Email: ${ }^{*}$ iqbalsurg@rediffmail.com

Received 27 December 2014; accepted 18 January 2015; published 22 January 2015

Copyright (C) 2015 by authors and Scientific Research Publishing Inc.

This work is licensed under the Creative Commons Attribution International License (CC BY).

http://creativecommons.org/licenses/by/4.0/

(c) () Open Access

\section{Abstract}

Background: The experience of short term results of laparoscopic inguinal hernia repair using 3D mesh in a developing country is reviewed. Methods: From January 2012 to February 2014, 53 patients underwent laparoscopic inguinal hernioplasty. A retrospective case series of 53 consecutive patients undergoing TEP/TAPP by a single surgical team was followed prospectively with a focused physical examination and interview. 4 out of 53 patients had recurrent hernia following open repairs and 49 had primary hernias. Data collected included operative time, intraoperative bleeding, intraoperative difficulties, immediate postoperative pain, chronic groin pain, recurrence, sensory disturbance, activity or occupational limitation and personal satisfaction. Results: All the patients were male aged 32 to 75 years with a mean age of 53.5 years. Mean operative time was 37.4 minutes; intraoperative dissection, blood loss were less; and immediate postoperative pain was negligible as assessed by VAS. There was no mortality or major morbidity. Mean follow-up was 12 months ( 2 to 18 months). Follow-up was completed by interview and physical examination. Hernia was not found to recur during the follow up period. Chronic pain occurred in 2 patients (3.7\%), which was mild in nature. Ninety-seven percent of patients were satisfied with their repair and would or had recommended TEP/TAPP to others using 3D Mesh. Conclusions: Short-term results of TEP/TAPP hernia repair using 3D mesh demonstrated to be an effective and safe procedure with low prevalence of chronic pain that is generally of a mild, infrequent nature. It was also concurred that there is decrease in operative time. Manipulation of mesh was significantly reduced. Intraoperative bleeding and use of post operative analgesia was reduced considerably. There was no recurrence, however the cost of the mesh increased the overall cost of the procedure acting as a limiting factor in a developing country.

\footnotetext{
*Corresponding author.
} 


\title{
Keywords
}

\author{
Inguinal Hernia, 3D Prolene Mesh, Totally Extra-Peritoneally (TEP), Trans-Abdominal \\ Pre-Peritoneal (TAPP)
}

\section{Introduction}

Inguinal hernias have plagued mankind for its upright gait since evolution. Around $16 \%$ of the patients presenting to our outpatient department have inguinal hernias. Various procedures like use of patients own tissues or prosthetic meshes have been used in the past with varying degrees of success. The revolutionary success of laparoscopic cholecystectomy has resulted in an intense effort to apply this concept of minimally invasive surgery to other operative procedures like inguinal hernia. The laparoscopic hernia surgery was initially a controversial topic with various studies publishing contradictory results. Unfortunately, the initial enthusiasm in laparoscopic hernioplasty was met with a disappointing early recurrence rate, which was as high as $25 \%$ in some series. However, with two decade of experience in lap hernia surgery, the dust seems to be settling down more towards accepting the superiority of the laparoscopic repairs over conventional open repairs. This is mainly due to the proper understanding of endoscopic inguinal anatomy, abandoning of initial procedures like plug and patch technique, refinement of the surgical techniques, introduction of the preperitoneal placement of mesh, etc.

Laparoscopic hernioplasty has several advantages over its open counterparts. First and foremost aspect from the patient's point of view is the reduced postoperative pain and short recovery period. Second, the entire myopectineal orifice can be inspected, allowing for repair of any unexpected concomitant hernias thereby reducing the chance of recurrence. Third, laparoscopic hernioplasty avoids previous operative scar site in patients with recurrent hernias [1]. In addition, simultaneously bilateral inguinal hernias can be managed conveniently by the same ports.

The disadvantages of laparoscopic repair include the need of general anaesthesia, the breach of peritoneum in TAPP repairs and the cost of the procedure and the need of expertise of the operating surgeon. The manipulation of the mesh in the confined space is quite tedious and prevents the operating surgeons from using laparoscopic technique in majority of the patients having inguinal hernias.

Recently a contoured anatomical 3D mesh has been introduced in the market. This mesh made of polypropylene resembles the normal inguinal area curvature. It has memory in the individual fibres of the polypropylene which results in even and quick placement of the mesh in the preperitoneal space. The marks on the medial aspect allow the surgeon to correctly orient the mesh covering the entire myopectineal orifice.

\section{Aim}

The aim of the present study is to assess the short-term results of TEP/TAPP using 3D mesh in a developing nation.

\section{Methods}

The records of 53 consecutive patients who had undergone lap inguinal (TEP/TAPP) hernioplasty between January 2012 and February 2014 at Department of Surgery, Govt. Medical College Srinagar, were reviewed and the patients recalled for clinical assessment. 49 patients had primary hernias and 4 had recurrent hernias following open hernia repairs. All procedures were performed by a single surgical team. The standard procedure in TEP, previously described, [2] was performed under general anesthesia with creation of sub-umbilical port, indigenously made balloon dissection and subsequent carbon dioxide insufflation of the preperitoneal space. Reduction of hernia sac was achieved using two $5 \mathrm{~mm}$ lower midline working ports. In cases of large hernias, the sac was ligated by 2.0 Polyglactin 910 thread and the distal sac was allowed to retract into the scrotum. Prefabricated polypropylene 3D mesh was then inserted in the extraperitoneal space covering the hernia orifices as shown in Figure 1. No fixation of the mesh was used.

Standard technique of TAPP was used in cases of larger, irreducible hernias or recurrent hernias. One $10 \mathrm{~mm}$ umbilical port for telescope and two $5 \mathrm{~mm}$ ports in both illac fossa were used for dissection. 


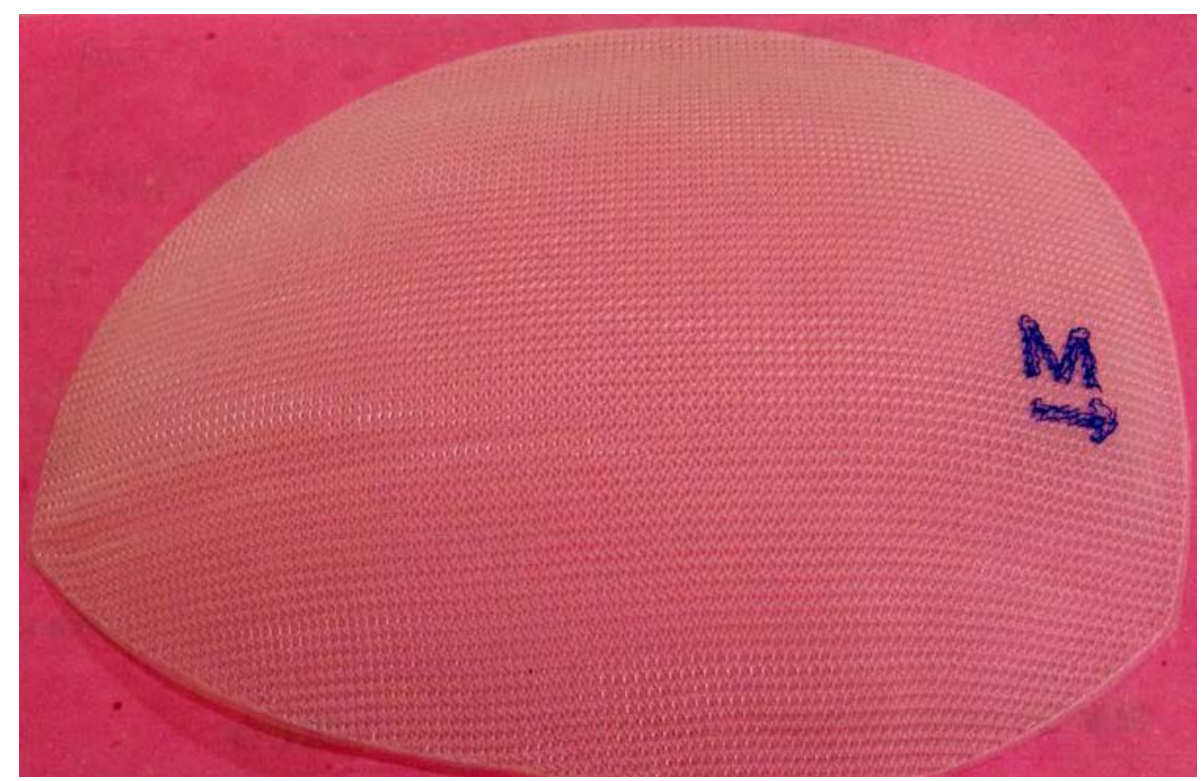

Figure 1. 3D mesh.

Specific issues addressed by the review were operative time, intra-operative dissection, intra-operative bleeding, immediate post-operative pain which was assessed by Visual Analogue Scale (VAS), chronic groin pain, tenderness, sensory disturbance, activity restriction or occupational limitation, and personal satisfaction. Specific examination to detect early recurrence was conducted in follow up period.

Chronic pain was defined in line with the International Association of Study of Pain as that pain persisting beyond 3 months from the date of surgery [3]. The type of pain (somatic ligamentous, neuropathic or visceral) and its severity were classified according to Cunningham [4]. Mild pain was defined as occasional pain or discomfort and caused no limitation to usual activities and allowed for a full return to pre-hernia lifestyle. Moderate pain was defined as pain interfering with or preventing a full return to pre-hernia activities such as sports, but not limiting basic daily activities. Severe pain was defined as pain that incapacitated the patient at regular intervals or interfered with basic activities of daily living such as walking, or caused significant occupational difficulties. Sensory impairment was categorized into anesthesia, paraesthesia or hyperesthesia.

\section{Results}

In the period January 2012 to February 2014, 53 inguinal hernias were repaired in 53 patients. We had no cases of bi-lateral hernias. Mean operative time was 37.4 minutes ranging from 27 to 80 minutes. Intraoperative dissection and intraoperative blood loss both were significantly less. One patient (1.8\%) experienced severe immediate postoperative pain which settled with routine analgesia. Most of the patients 47 (88.67\%) were discharged within 24 hours of surgery. The mean length of follow-up was 12 months (range 2 - 18 months). There was no recurrence (0\%). Two patients (3.7\%) described persistent chronic pain which was mild in nature. No patients had ongoing moderate or severe pain. In one of these, pain type was somatic ligamentous, localized to the pubic tubercle. One patient had neuropathic pain presumably from irritation of local nerves. Three patients (5.6\%) had groin tenderness on examination. Tenderness was located over the pubic tubercle in 1 patient, over the deep ring in 1 patient and in the spermatic cord at the top of the scrotum in 1 patient. One patient had sensory impairment (1.88\%). This patient had a small area of anesthesia in the groin medially. No patients had paraesthesia or hyperesthesia. No patients had testicular atrophy, although one patient had a tethered cord resulting in an elevated testicle. 2 patients developed seroma in the distal part of the sac which settled with conservative management. No serious complications following laparoscopic inguinal hernioplasty were experienced. 51 patients (96.22\%) were either satisfied or extremely satisfied with their repair and would, or had already recommended TEP/TAPP to a friend Four patients had previously experienced an open repair and all felt that their TEP/TAPP repair was associated with less pain and shorter time to return to usual activities. 
Untoward events during and after the procedure.

\begin{tabular}{cc}
\hline Severe immediate post operative pain & $1(1.88 \%)$ \\
Groin tenderness & $3(5.65 \%)$ \\
Seroma & $2(3.77 \%)$ \\
Sensory impairment & $1(1.88 \%)$ \\
Tethering of cord & $1(1.88 \%)$ \\
Chronic pain & $2(3.77 \%)$ \\
\hline
\end{tabular}

\section{Discussion}

This ongoing study is being conducted in department of general surgery govt medical college srinagar located in the extreme north of India. This paper documents the early results of laparoscopic groin hernia repair using 3D mesh (TAPP/TEP).

All the patients were male, aged between 32 to 75 years with a mean age of 53.5 years. The lower incidence of immediate post-operative pain observed in our study can be explained on the fact that pain is a very subjective experience, as the pain thresholds varies significantly among various individuals, different races and ethnicities, different sexes, so what can be a painful stimulus for one person may not be perceived as painful for another person [5].

Another factor responsible for reduced post-operative pain observed in this study is the fact that using a 3D mesh eliminates the necessity to fix the mesh either with sutures or tacks in TEP as is needed with a flat mesh thereby avoiding nerve entrapment [6]. However, the harvested peritoneal fold in TAPP is closed by sutures. The reduced post-operative pain noted in our study has been verified as a major advantage of laparoscopic hernia repair by other studies that were conducted recently [7] [8].

The 3D mesh because of its memory and 3D configuration was found to be easier to handle thereby reducing intraoperative time, after all a flat sheet of mesh is not an ideal configuration for laparoscopic repair as the inguinal anatomy is anything but the two-dimensional image as seen on the monitor thus the 3D configuration of the mesh which is anatomically formed and shaped to the inguinal anatomy is ideal thus making us recommend the 3D mesh to be used by surgeons especially beginners to minimize the stress [9]. Also a major advantage of laparoscopic hernia repair is in cases of bilateral inguinal hernias, where laparoscopy allows for both hernias to be repaired in a single operation without need for additional ports or incisions [10].

No recurrence at 18 months following TEP/TAPP occurred in our series whereas in other study the recurrence tended to be a late phenomenon, occurring at a median of 36 months which supports the need for long-term follow-up studies to accurately determine the success rate of surgery. One of the flaws of studies that compare recurrence of laparoscopic versus open repairs is that they unfairly compare the results of an operation that most surgeons are highly experienced at, with those of a relatively new technique that has variable levels of surgeon experience. An appropriate comparison would be that which compares the best results achievable with both techniques. An example of this problem is well illustrated by the recently published Veterans Affairs cooperative study comparing TEP with the Lichtenstein open repair, which concluded against laparoscopic repair on the basis of higher overall recurrence [7].

However, when the TEP experience of individual surgeons was analyzed, it was found that surgeons experienced in TEP had a recurrence rate of $<5 \%$ that was equal to that achieved by the Lichtenstein arm. Others have shown in large multicentre randomized trials that TEP has a lower recurrence risk than open repairs [11]. The importance of follow-up by physical examination was highlighted in the present study by no recurrence in the series, however at present it is too early to comment regarding recurrence. Most published hernia studies conduct follow-up only by telephone or written questionnaire. These methods of follow-up may not be accurate [12]. It is noteworthy that in contrast to the recommendations of the UK National Institute for Clinical Excellence (NICE) [13] the incidence of recurrence (0\%) in the present series is rather low, reflecting our philosophy that TEP/TAPP is the preferred method of repair of all groin hernias, including first-time repairs by experienced surgical team using 3D mesh.

Chronic pain in the post operative follow up is a major concern in laparoscopic repairs. Although ongoing chronic pain complicated $3.7 \%$ of the present series TEP/TAPP repairs, it was in almost all cases of a mild and 
occasional nature that allowed a full return to pre-hernia activities. We were stringent in the application of our pain definitions and included patients who experienced any groin pain, regardless of how minor or infrequent. The corollary of this is that $96 \%$ of the present patients were completely free of any long-term groin pain whatsoever. Many studies of various open repairs, including tension-free repairs, report both a higher rate and greater severity of chronic pain.

This is exemplified by the Cooperative Hernia Study which, using the same pain definitions, found 53\% of patients had pain, being moderate or severe in $10 \%$, at 2 years following open repairs [4].

One large review of $>40$ hernia studies including all major repair techniques demonstrated that laparoscopic TEP repairs had the lowest prevalence of chronic pain [14].

Indeed, a recent British prospective randomized trial showed that patients who had undergone Lichtenstein repairs had a fivefold higher risk of chronic pain when compared to those who have had laparoscopic repairs. [15].

Patients who underwent open herniorrhaphies are also twice as likely to seek the help of a pain specialist [16]. The pain following TEP/TAPP is most commonly somatic ligamentous, which is thought to arise from inflammation of the ligamentous insertions around the pubic tubercle, perhaps associated with fixation. This differs from the type of pain usually seen following open repairs, which is usually neuropathic, resulting from nerve irritation. This difference is explained readily because dissection in TEP/TAPP occurs in a different plane to the ilioinguinal and iliohypogastric nerves, which, in contrast, are vulnerable to injury in an open repair. This concept is further supported by the low prevalence of sensory impairment in the present series of laparoscopic repairs. If the somatic pain observed following TEP/TAPP relates to fixation, then alternatives to staple fixation such as tissue glue or non-fixation may further reduce chronic pain [17]. Such alternatives, however, would require further investigation to ensure that reduced fixation does not come at the expense of increased recurrence. Two randomized trials have demonstrated no difference in recurrence rates and postoperative pain after repairs with fixation versus no fixation, suggesting that fixation may not be necessary and also that it does not contribute to chronic pain. Fibrin glue may also be used to fix mesh [8].

Further the unique shape of 3D mesh is designed to conform to the inguinal anatomy, contour minimizes buckling \& design reduces the need for fixation [18]. Sealed edge \& medial orientation marker facilitate accurate placement \& positioning. But the cost of 3D mesh is twice than that of flat mesh. Elimination of tack fixation of 3D mesh during TEP significantly reduces use of postoperative analgesia and hospital length of stay without increasing rate of recurrence [19].

\section{Conclusions}

Laparoscopic inguinal mesh hernioplasty using 3D mesh is a safe and efficacious method of hernioplasty with no recurrence in early post-operative period and negligible pain.

Surgeons experienced in TEP/TAPP should be encouraged to report their long-term experience so that the true potential of this technique, rather than just learning curve results, is better understood. However, the cost of the $3 \mathrm{D}$ mesh is greater than flat mesh which is a major limiting factor in the developing world.

\section{References}

[1] Palanivelu, C. (2007) Art of Laparoscopic Surgery. Jaypee Brothers, New Delhi.

[2] Kuthe, A., Saemann, T., Tamme, C., et al. (1998) Technique of Total Extraperitoneal Endoscopic Hernioplasty of Inguinal Canal. Zentralbl Chir, 123, 1428-1435.

[3] Bruce, J.P., Balan, A.S., Smith, W.C.S., et al. (2004) Quantitative Assessment of Chronic Post Surgical Pain Using McGill Pain Questionnaire. The Clinical Journal of Pain, 20, 70-75. http://dx.doi.org/10.1097/00002508-200403000-00002

[4] Cunningham, J., Temple, W.J., Mitchell, P., et al. (1996) Operative Hernia Study. Pain in the Post Operative Patient. Annals of Surgery, 224, 598-602. http://dx.doi.org/10.1097/00000658-199611000-00003

[5] Melzack, R. and Wall, P.D. (1996) The Challenge of Pain. 2nd Edition, Penguin Books, New York, 17-19.

[6] Ferzli, G.S., et al. (1999) Prospective Randomized Study of Stapled versus Unstapled Mesh in a Laparoscopic Preperitoneal Inguinal Hernia Repair. Journal of the American College of Surgeons, 188, 461-465. http://dx.doi.org/10.1016/S1072-7515(99)00039-3 
[7] Neumayer, L., Giobbie-Hurder, A., Jonasson, O., et al. (2004) Open Mesh versus Laparoscopic Mesh Repair of Inguinal Hernia. The New England Journal of Medicine, 350, 1819-1827. http://dx.doi.org/10.1056/NEJMoa040093

[8] Carter, J. and Duh, Q.Y. (2011) Laparoscopic Repair of Inguinal Hernias. World Journal of Surgery, 35, $1519-1525$. http://dx.doi.org/10.1007/s00268-011-1030-x

[9] (1998) Laparoscopic Groin Hernia Repair Using a Curved Prosthesis without Fixation. Le Journal de Celio-Chirurgie, 28, 64-68.

[10] Wauschkuhn, C., Schwarz, J., Boekeler, U., et al. (2010) Laparoscopic Inguinal Hernia Repair: Gold Standard in Bilateral Hernia Repair? Results of More than 2800 Patients in Comparison to the Literature. Surgical Endoscopy, 24, 3026-3030. http://dx.doi.org/10.1007/s00464-010-1079-X

[11] Liem, M.S.L., van der Graaf, Y., van Steensel, C.J., Boelhouwer, R.U., Clevers, G.-J., Meijer, W.S., et al. (1997) Comparison of Conventional Anterior Surgery and Laparoscopic Surgery for Inguinal Hernia Repair. New England Journal of Medicine, 336, 1541-1547. http://dx.doi.org/10.1056/NEJM199705293362201

[12] Herzog, U. (1990) Late Results Following Inguinal or Femoral Hernia Surgery. Langenbecks Archiv für Chirurgie, 375, 5-10. http://dx.doi.org/10.1007/BF00186114

[13] National Institute for Clinical Excellence (2001) Guidelines for the Use of Laparoscopic Surgery for the Treatment of Inguinal Hernias in the NHS. National Institute for Clinical Excellence, London.

[14] Poobalan, A.S., Bruce, J., Smith, W.C., King, P.M., Krukowski, Z.H. and Chambers, W.A. (2003) A Review of Chronic Pain after Inguinal Herniorrhaphy. Chinese Journal of Pain, 19, 48-54.

[15] Douek, M., Smith, G., Oshowo, A., Stoker, D.L. and Wellwood, J.M. (2003) Prospective Randomised Controlled Trial of Laparoscopic versus Open Inguinal Hernia Mesh Repair: Five Year Follow up. British Medical Journal, 326, 10121013. http://dx.doi.org/10.1136/bmj.326.7397.1012

[16] Hindmarsh, A.C., Cheong, E., Lewis, M.P. and Rhodes, M. (2003) Attendance at a Pain Clinic with Severe Chronic Pain after Open and Laparoscopic Inguinal Hernia Repairs. British Journal of Surgery, 90, 1152-1154.

[17] Khajanchee, Y.S., Urbach, D.R., Swanstrom, L.L. and Hansen, P.D. (2001) Outcomes of Laparoscopic Herniorrhaphy without Fixation of Mesh to the Abdominal Wall. Surgical Endoscopy, 15, 1102-1107.

[18] Bell, R.C.W. and Price, J.G. (2003) Laparoscopic Inguinal Hernia Repair Using Anatomically Contoured Three Dimensional Mesh. Surgical Endoscopy, 17, 1784-1788. http://dx.doi.org/10.1007/s00464-002-8763-4

[19] Koch, C.A., Greenlee, S.M., Larson, D.R., Harrington, J.R. and Farley, D.R. (2006) Randomized Prospective Study of Totally Extraperitoneal Inguinal Hernia Repair: Fixation versus No Fixation of Mesh. Journal of the Society of Laparoendoscopic Surgeons, 10, 457-460. 
Scientific Research Publishing (SCIRP) is one of the largest Open Access journal publishers. It is currently publishing more than 200 open access, online, peer-reviewed journals covering a wide range of academic disciplines. SCIRP serves the worldwide academic communities and contributes to the progress and application of science with its publication.

Other selected journals from SCIRP are listed as below. Submit your manuscript to us via either submit@scirp.org or Online Submission Portal.
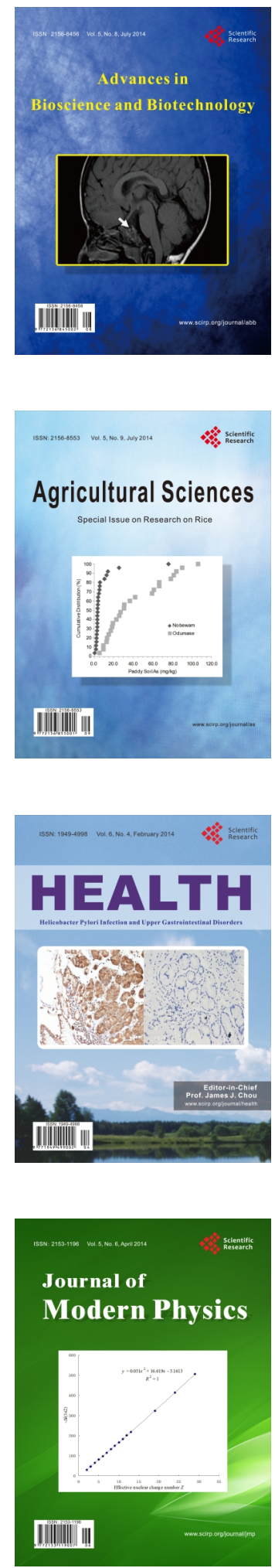
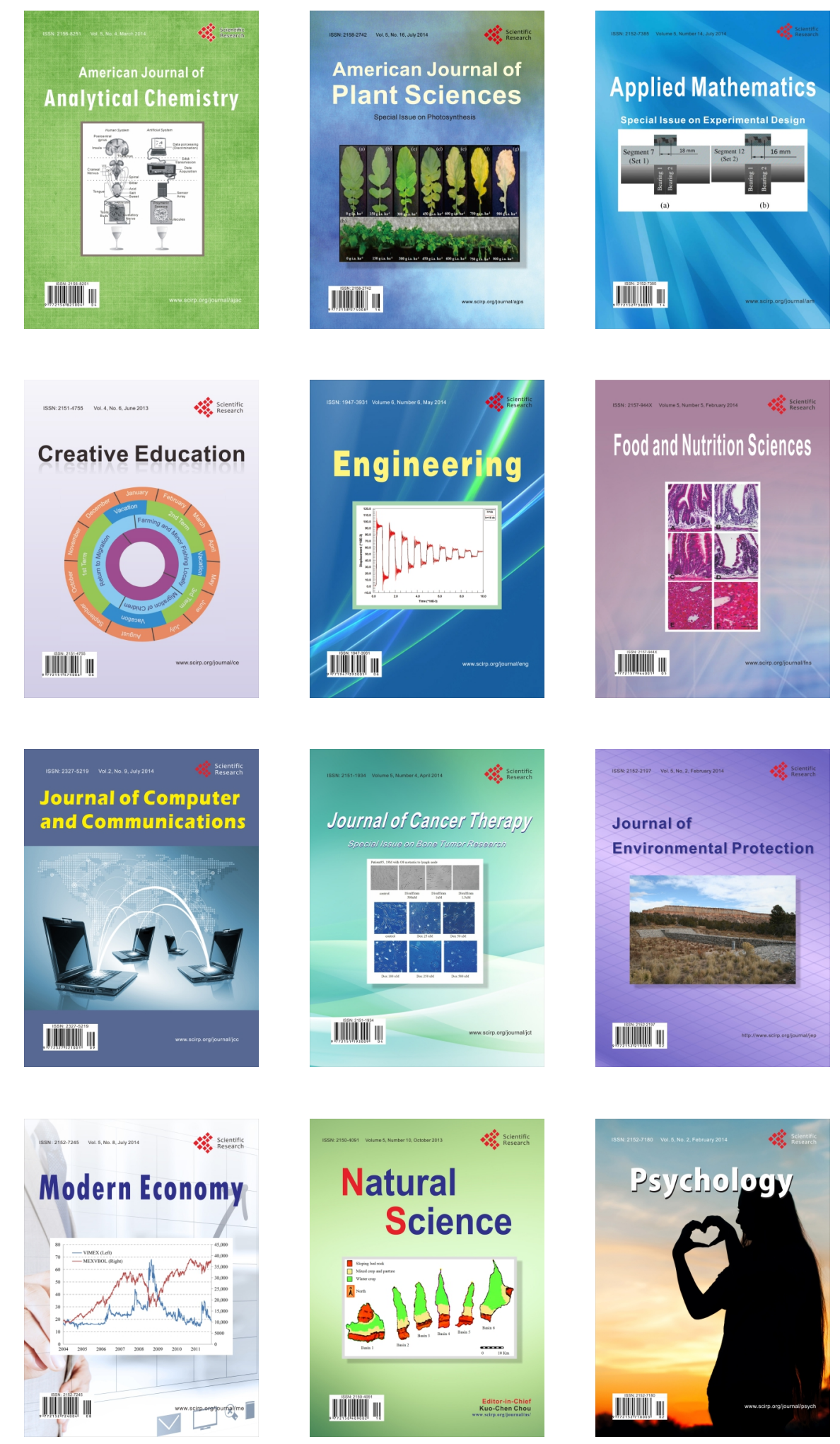